\title{
Emittance Measurements of the 4.5 MeV UCLA RF Photo-Injector*
}

\author{
S. C. Hartman, N. Barov, S. Park, \\ C. Pellegrini, J. Rosenzweig, G. Travish, R. Zhang \\ Department of Physics, University of California, Los Angeles 90024 \\ P. Davis, C. Joshi, G. Hairapetian \\ Electrical Engineering Department, University of Califomia, Los Angeles 90024
}

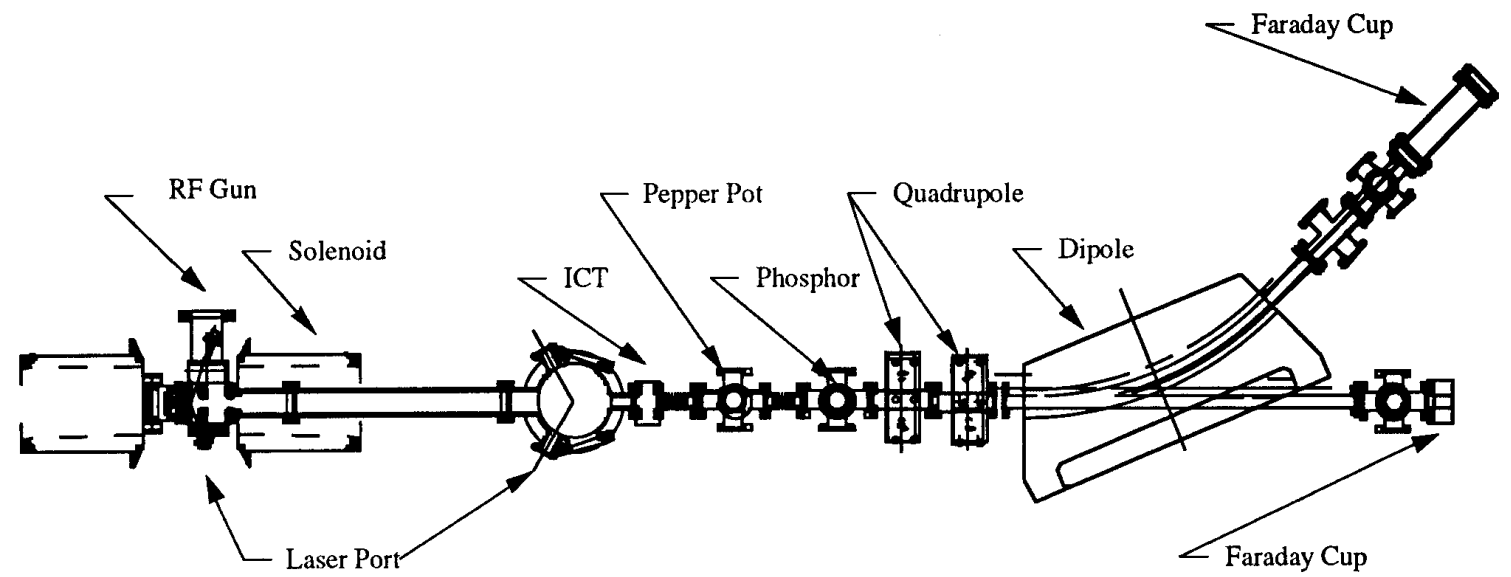

Figure 1.

Abstract

The 1.5 cell RF photo-injector $(1,2)$ has been operated for the past several months using a copper cathode illuminated by 4 ps long pulses of UV $(266 \mathrm{~nm})$ light, with a variable energy of between 0 to $300 \mu \mathrm{J}$. This typically produces up to $3 \mathrm{nC}$ of charge per bunch. Because space charge forces dominate the electron beam transport a pepper pot measurement system is used to measure the emittance. The emittance is measured as a function of charge, peak accelerating field, laser spot size and initial phase with respect to the RF field. This is accomplished with an automated control and data acquisition system which can measure single shot emittances at a rate of $5 \mathrm{~Hz}$ developed at UCLA. The experimental results obtained are then compared with theory and simulations.

\section{INTRODUCTION}

The UCLA photo-injector produces a very high peak current beam, up to 250 amps, at relatively low energy, $4.5 \mathrm{MeV}$. The consequence of this is that electron beam transport is in the highly non-linear space charge dominated regime. Since the beam is space charge dominated, linear beam transport formalisms such as matrix transformations do not apply. This means that the usual quadrupole scan technique is not valid for the UCLA photo-electron beam. To overcome this problem a pepper pot emittance measurement apparatus has been designed and implemented. The pepper pot is designed such that upon the electron beams passage through the pepper pot it is transformed from a space charge dominated beam to an

*Work Supported by SDIO/IST through ONR Grant No. N0001490-J-1952 and US DOE Grant DE-FG03-92ER-40493 emittance dominated beam. Once the electron beam is in the emittance dominated regime one can use linear transform theory to calculate the emittance. Because of pulse to pulse fluctuations in the beam charge the measurement must be made single shot. All the beam parameters are measured every shot via fast sample and hold electronics.

\section{EXPERIMENTAL DESIGN}

\section{A. RF Photo-Injector and Drive Laser}

The UCLA photo-injector is a one and half cell standing wave accelerator operating in the $\pi$-mode with peak accelerating fields of the order of $100 \mathrm{MV} / \mathrm{m}$. The photocathode is illuminated by frequency chirped, pulse compressed, frequency quadrupled Nd-YAG laser. This laser system delivers $0-300 \mu \mathrm{J}$ of energy on the cathode in less than $4 \mathrm{ps}$ and can be injected on axis or at a $70^{\circ}$ angle of incidence.

\section{B. Diagnostics}

Figure 1 shows the beam line configuration and the location of the diagnostics used in this experimet. Phosphor screens are employed at three locations to measure spot size and emittance patterns. The phosphors used were experimentally confirmed to be linear so no distortions in spot size are due to non linear phosphor response. Faraday cups are used to measure the charge and some of the phosphor screens are floating and can double as faraday cups. This way one can gather image and charge data simultaneously. To measure the charge when taking emittance measurements we use an Integrating Current Transformer, ICT, which measures the total charge which passes through it. Because it is designed to 
respond to time scales of the order of ps it automatically ignores the dc like dark current background.

\section{PEPPER POT AND DATA ACQUISITION}

\section{A. Pepper Pot Considerations}

The pepper pot used in these experiments is actually an array of eight slits. After the electron beam traverses the slits it drifts approximately $20 \mathrm{~cm}$ onto a phosphor screen. The image then gives the full phase space, emittance, and other beam parameters. The width of the slit was chosen by considering the envelope equation, eq. 3.1. For a round beam $A$ is the normalized beam transverse dimension, $\beta$ is the normalized velocity, $\gamma$ is the normalized energy, and $\varepsilon$ is the normalized emittance.

$$
A^{\prime \prime}-\frac{\beta \gamma k}{A}-\frac{\varepsilon_{n}^{2}}{A^{3}}=0 \quad \text { where } \quad \mathrm{A}=\mathrm{a}[\beta \gamma]^{1 / 2}
$$

Taking the ratio of the space charge to emittance term yields,

$$
\Re=\frac{2 a^{2} I_{p}}{(\beta \gamma)^{1 / 2} \varepsilon_{n}^{2} I_{A}}
$$

where $I_{p}$ is the peak electron beam current and $I_{a}$ is the Alfven current of $17 \mathrm{kA}$. When this ratio is greater than unity space charge forces dominated the electron beam and when the ratio is less than unity emittance effects dominates the electron beam transport. By choosing the slit widths wisely we can go from the space charge dominated regime to the emittance dominated regime. Now if we scale the transverse dimension by $\eta$,

$$
I_{p}^{\prime}=\eta^{2} I_{p} ; \quad a^{\prime}=\eta a ; \quad \varepsilon_{n}^{\prime}=\eta \varepsilon_{n}
$$

the ratio of the space charge term to the emittance term becomes.

$$
\mathfrak{R}^{\prime}=\mathfrak{R} \eta^{2}
$$

Using this expression and plugging in the numbers for the UCLA experiment we get $\eta=0.01$ which corresponds to a slit size of $50 \mu \mathrm{m}$.

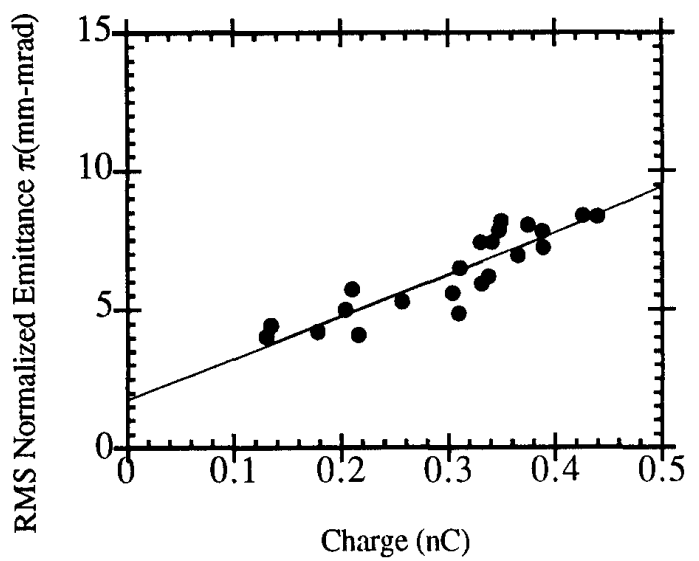

Figure 2.

\section{EMITTANCE MEASUREMENTS}

\section{A. Definition of Emittance}

Electron beams do not typically have sharp boundaries so the definition of emittance is ambiguous at times. Some laboratories define emittance for a certain percentage of the beam particles enclosed within an ellipse, commonly $63 \%$ or $90 \%$ (3). For the following measurements we define our emittance as the root-mean-square emittance given by

$$
\varepsilon_{r m s}=\left(\left\langle x^{2}\right\rangle\left\langle x^{\prime 2}\right\rangle-\left\langle x \cdot x^{\prime}\right\rangle^{2}\right)^{1 / 2}
$$

and the normalized emittance is given by

$$
\varepsilon_{n}=\beta \gamma \varepsilon_{r m s}
$$

\section{Emittance Vs Charge}

The electron beam emittance in all of the following was done with a beam momentum of $3.5 \mathrm{MeV} / \mathrm{c}$. The first step in measuring the emittance is to measure the linear increase as a function of charge. The laser energy delivered to the cathode was varied and the emittance was measured as a function of charge transported through the ICT and to the slits. For all other parameters fixed one expects the emittance to be linear in charge as in the following equation(4)

$$
\varepsilon_{x}^{s c}=\frac{\pi}{4} \frac{1}{\alpha \kappa} \frac{1}{\sin \left(\vartheta_{0}\right)} \frac{I}{I_{A}} \mu
$$

Each emittance verses charge plot takes approximately thirty to sixty seconds to complete. So one can adjust on-line the

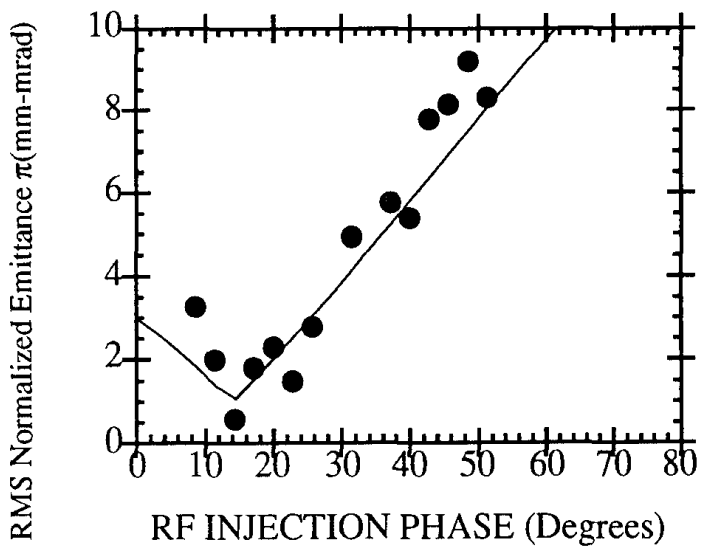

Figure 3.

beam parameters to minimize the electron beam emittance. This is a very powerful diagnostic.

\section{RF induced Emittance Vs Laser Injection Phase}

To measure the $\mathrm{rf}$ induced emittance verses laser injection phase many emittance verse charge data runs were taken with different laser injection phases. The procedure for extracting the If induced emittance is as follows. For each laser injection phase the data is plotted and fit to a line. The slope and intercept of this line are then extracted. The zero charge intercept gives the rf induced emittance. The following graph 
is a plot of the zero intercepts of many rf injection phases. The data is then fit to the equation(4)

$$
\varepsilon_{x}^{f f}=\alpha \kappa\left\langle x^{2}\right\rangle \sqrt{\left\langle(\Delta \phi)^{2}\right\rangle}|\cos \langle\phi\rangle|
$$

Notice that the data has the correct scaling as one would expect.

\section{E. Emittance Vs Peak Accelerating Field}

To explore the effect of the variation of the peak electric field the rf power into the photoinjector was varied. Since the final beam energy is proportional to the peak accelerating field we recover the expected linear decreasing of the emittance.

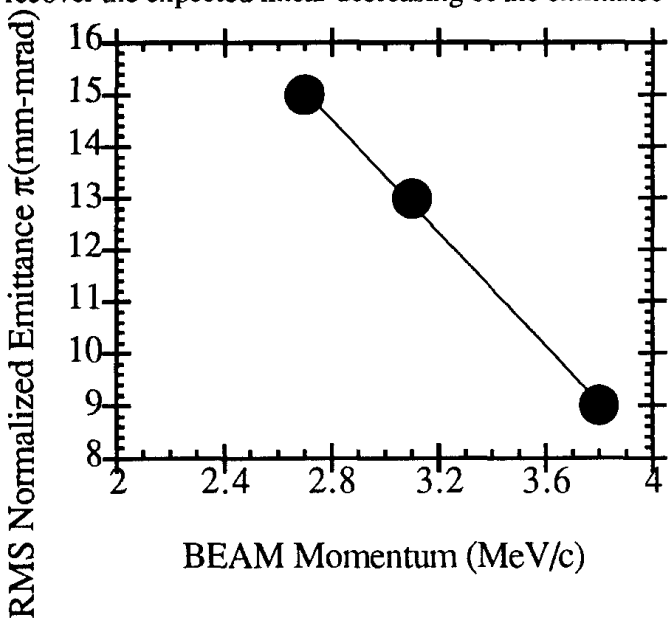

Figure 6

\section{F. Rf Injection Phase Calibration}

The beam energy verse if injection phase was measured in order to calibrate the absolute phase. The equations of motion were then integrated and compared to the experiment. This data highlights the fact that the rf gun was unbalanced. The field in the full cell was 1.8 times that in the half cell. This was confirmed with a bead pull experiment after the gun was removed for a postmortem cold test.

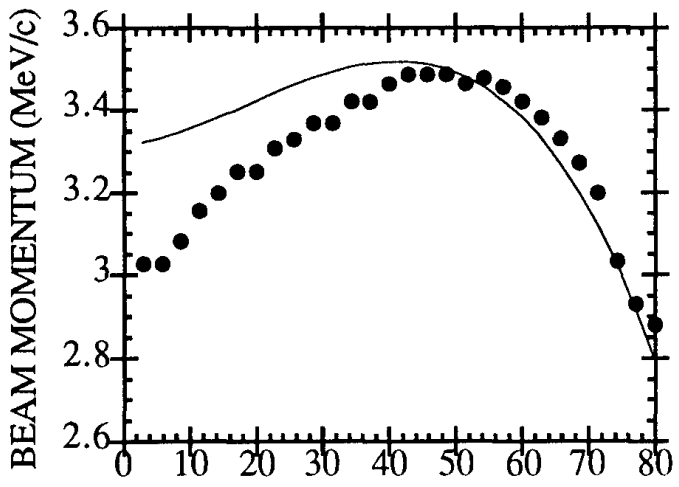

RF Phase (Deg)

\section{DISCUSSION}

The emittance measurements presented above are the first round results and suffer from a few problems. The first is that upon installation of the photo-injector the micrometer which holds the cathode in place was bumped. This caust ' the gun to become detained. The gun was then retuned on-line by adjusting the cathode position until the shunt impedance was maximized. Since the shunt impedance of the full cell is larger than the half cell the peak fields in the full cell were favored. The second artificial emittance growth mechanism was that the phosphor screens which were use to measure the spots were at $45^{\circ}$ angles with respect to the beam. This enlarged the spot size artificially and due to short depth of focus induced some parallax broadening and blurring of the image. These problems were eliminated in a subsequent attempt to re measure the emittance but due to time limitations the measurements were not completed in time for this conference. The emittance values given are thus a set of worse case values.

\section{CONCLUSION}

The UCLA photo-injector has been operated successfully. The measurements show that the emittance scales as expected. The important thing to note is that for these set of emittance runs the rf photo-injector had a field imbalance. The filled in the full cell was 1.8 times that in the half cell. This contributed to emittance blowup as did the phosphor screens used to measure the emittance. The phosphor screens were placed onto the beamline at a $45^{\circ}$ angle. This created broadening of the line widths

\section{References}

1. S. C. Hartman et al., Photocathode Driven Linac at UCLA for FEL and Plasma Wakefield Acceleration Experiments, Particle Accelerator Conference Sanfransisco, CA., 1991), pp. 2967.

2. K. Batchelor et al, European Particle Conference Accelerator Conference Rome, Italy, June 7-12, 1988),

3. C. Lejeune, J. Aubert, Eds., Emittance and Brightness Definitions and Measurements (Academic, New York, 1980).

4. K.-J. Kim, Nuclear Instruments and Methods in Physics Research A275, 201-218 (1989).

Figure 5 